\title{
Incorporation of Kr and Xe in Uranium Mononitride; a Density Functional Theory Study
}

\author{
Lin Yang and Nikolas Kaltsoyannis*
}

Department of Chemistry, School of Natural Sciences, The University of Manchester, Oxford Road, Manchester M13 9PL

\section{Supporting Information}

Method for determining the chemical potential of $\mathbf{U}$ and $\mathbf{N}$ in $\mathbf{U N}$. According to the phase diagram of UN, nearstoichiometric UN should be bound by $\mathrm{U}$ rich condition (in equilibrium with bulk $\mathrm{U}$ ) and $\mathrm{N}$ rich condition (in equilibrium with $\left.\mathrm{U}_{2} \mathrm{~N}_{3}\right)$. Then the chemical potential of $\mathrm{U}\left(\mu_{\mathrm{U}}\right)$ and $\mathrm{N}\left(\mu_{\mathrm{N}}\right)$ should follow the following equation:

$$
E\left(\mathrm{U}_{x} \mathrm{~N}_{y}\right)=x \mu_{\mathrm{U}}+y \mu_{\mathrm{N}}
$$

in which $E\left(\mathrm{U}_{x} \mathrm{~N}_{y}\right)$ is the total energy of $\mathrm{U}_{x} \mathrm{~N}_{y}$ phase. From the PW91 functional, the total energies of bulk $\alpha-\mathrm{U}\left(\mathrm{U}_{4}\right)$, $\mathrm{UN}\left(\mathrm{U}_{4} \mathrm{~N}_{4}\right)$, and $\mathrm{U}_{2} \mathrm{~N}_{3}$ are $-44.60 \mathrm{eV},-88.58 \mathrm{eV}$, and $-54.46 \mathrm{eV}$, respectively. Applying these values to the above equation, the calculated $\mu_{\mathrm{U}}$ and $\mu_{\mathrm{N}}$ are: $-11.98 \mathrm{eV}<\mu_{U}<-11.15 \mathrm{eV}$ and $-10.99 \mathrm{eV}<\mu_{N}<-10.17 \mathrm{eV}$.

Table S1. Cutoff energy convergence test.

\begin{tabular}{cc}
\hline Cutoff energy /eV & Total energy /eV \\
\hline 520 & -88.5805 \\
550 & -88.5814 \\
580 & -88.5834 \\
610 & -88.5855 \\
640 & -88.5878 \\
\hline
\end{tabular}

Table S2. Supercell size convergence test.

\begin{tabular}{cc}
\hline Cutoff energy /eV & Total energy /eV \\
\hline $3 \times 3 \times 3$ & -709.1991674 \\
$4 \times 4 \times 4$ & -709.2048496 \\
$5 \times 5 \times 5$ & -709.2142592 \\
\hline
\end{tabular}


Table S3. Uncorrected defect formation energies $\left(E_{f}\right)$ and the elastic field correction term $\left(E_{e l}\right)$ for AFM UN.

\begin{tabular}{ccccc}
\hline & \multicolumn{3}{c}{$E_{f} / \mathrm{eV}$} & $E_{e l} / \mathrm{eV}$ \\
\cline { 2 - 4 } & $\mathrm{UN}_{1-x}$ & $\mathrm{UN}$ & $\mathrm{UN}_{1+x}$ & 0.00 \\
\hline $\mathrm{V}_{\mathrm{U}}$ & 3.17 & 2.75 & 2.34 & 0.00 \\
$\mathrm{~V}_{\mathrm{N}}$ & 1.76 & 2.18 & 2.59 & 0.04 \\
$\mathrm{FP}_{\mathrm{N}}$ & 4.92 & 4.92 & 4.92 & 0.20 \\
$\mathrm{SD}$ bound & 4.26 & 4.26 & 4.26 & 0.00 \\
$\mathrm{SD}$ unbound & 4.96 & 4.96 & 4.96 & \\
\hline
\end{tabular}

Table S4. Uncorrected defect formation energies $\left(E_{f}\right)$ and the elastic field correction term $\left(E_{e l}\right)$ for FM UN.

\begin{tabular}{ccccc}
\hline & & $E_{f} / \mathrm{eV}$ & \multirow{2}{*}{$E_{e l} / \mathrm{eV}$} \\
\cline { 2 - 4 } & $\mathrm{UN}_{1-x}$ & $\mathrm{UN}$ & $\mathrm{UN}_{1+x}$ & 0.00 \\
\hline $\mathrm{V}_{\mathrm{U}}$ & 3.43 & 3.01 & 2.60 & 0.00 \\
$\mathrm{~V}_{\mathrm{N}}$ & 1.90 & 2.31 & 2.72 & 0.01 \\
$\mathrm{FP}_{\mathrm{U}}$ & 9.46 & 9.46 & 9.46 & 0.00 \\
$\mathrm{FP}_{\mathrm{N}}$ & 5.04 & 5.04 & 5.04 & 0.60 \\
$\mathrm{SD}$ bound & 4.47 & 4.47 & 4.47 & 0.00 \\
$\mathrm{SD}$ unbound & 5.15 & 5.15 & 5.15 & \\
\hline
\end{tabular}

Table S5. N and U vacancy formation energies calculated $v s \mathrm{~N}_{2}$ molecule and $\mathrm{U}$ bulk $\left(E^{(1)} f\right)$, and isolated $\mathrm{N}$ and $\mathrm{U}$ atoms $\left(E^{(2)} f\right)$.

\begin{tabular}{|c|c|c|c|c|c|c|}
\hline & \multicolumn{3}{|c|}{$E_{f}^{(1)} / \mathrm{eV}$} & \multicolumn{3}{|c|}{$E_{f}^{(2)} / \mathrm{eV}$} \\
\hline & $\mathrm{VN}$ & VU & Magnetic order & $\mathrm{VN}$ & VU & Magnetic order \\
\hline \multirow{2}{*}{ Present work } & 4.42 & 3.17 & AFM & & & \\
\hline & 4.56 & 3.43 & FM & & & \\
\hline $\operatorname{Ref}[1]$ & 4.24 & 3.74 & Spin-polarization & 9.43 & 10.55 & Spin-polarization \\
\hline $\operatorname{Ref}[2]$ & 4.42 & 3.22 & FM & 9.56 & 10.39 & FM \\
\hline $\operatorname{Ref}[3]$ & & & & 7.81 & 6.89 & AFM \\
\hline $\operatorname{Ref}[4]$ & & & & 9.4 & 9.1 & $\begin{array}{c}\text { Neglect } \\
\text { spin-polarization }\end{array}$ \\
\hline
\end{tabular}




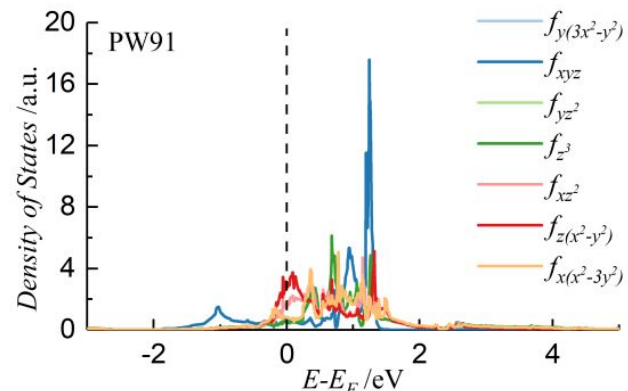

(a)

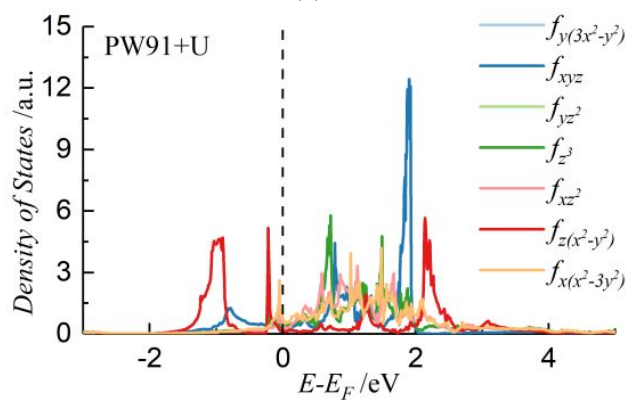

(c)

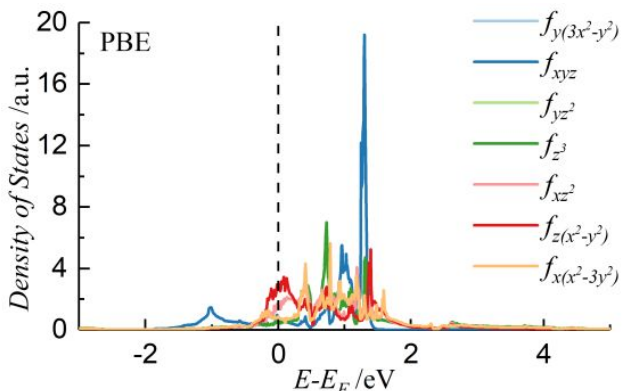

(b)

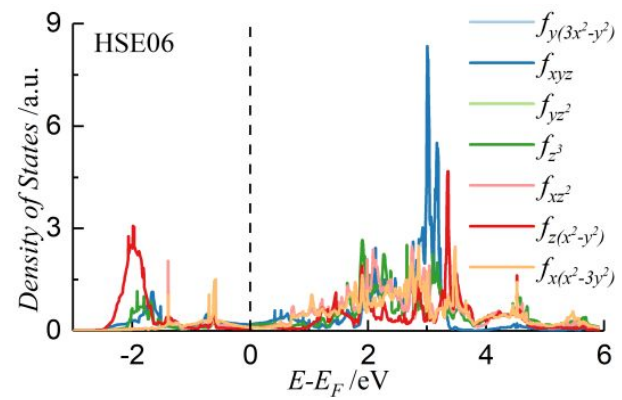

(d)

Figure S1. The partial density of states of the U f orbitals in AFM UN bulk obtained from PW91, PW91+U, PBE, and HSE06. Only the spin up states are shown as representation.

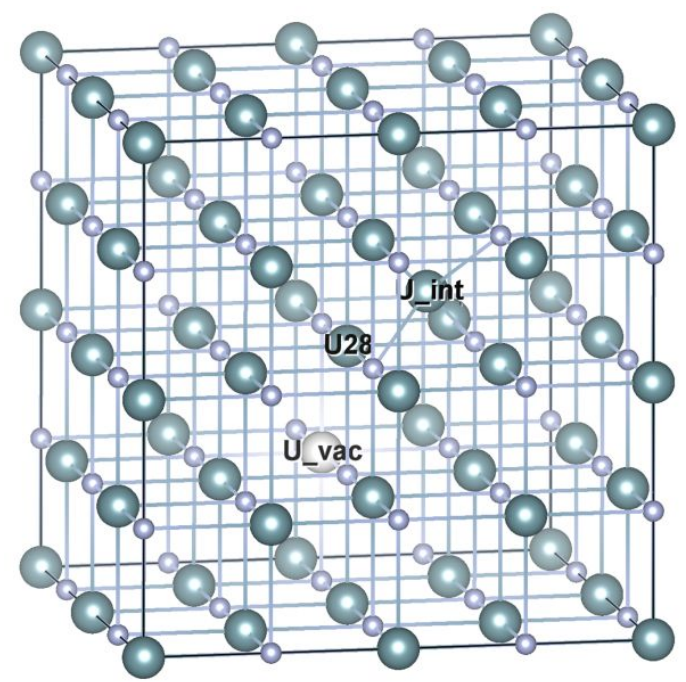

Figure S2. Unrelaxed UN supercell containing U Frenkel pair defect. 


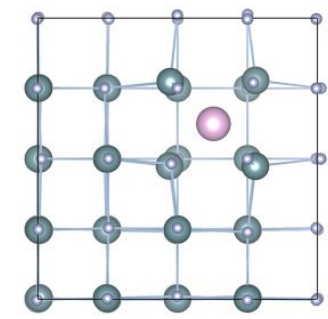

(a) Interstitial

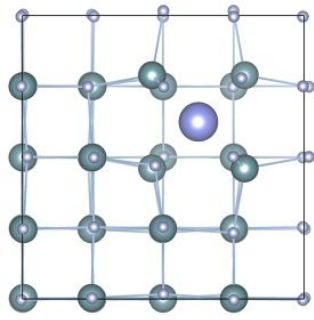

(a) Interstitial

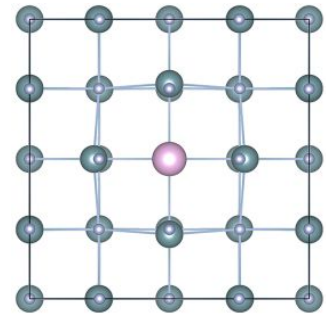

(b) $\mathrm{V}_{\mathrm{N}}$

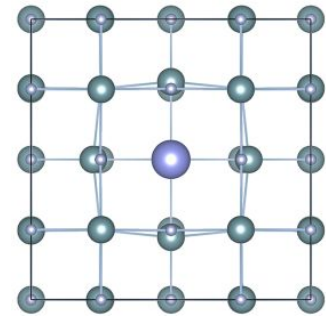

(b) $\mathrm{V}_{\mathrm{N}}$

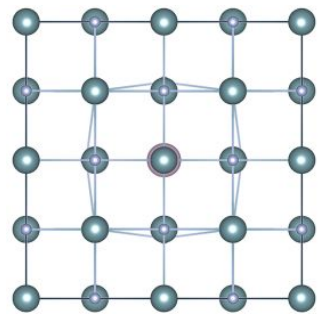

(c) $\mathrm{V}_{\mathrm{U}}$

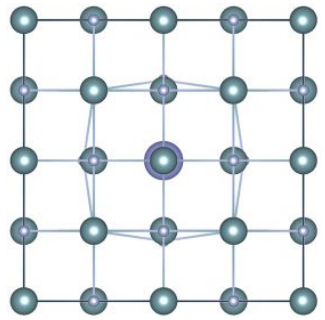

(c) $V_{U}$

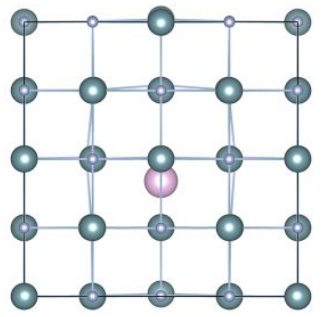

(d) $\mathrm{SD}$

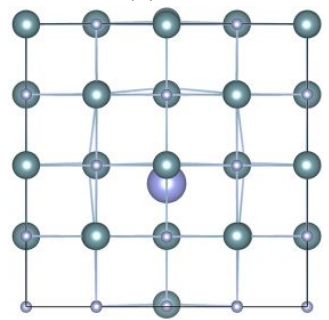

(d) SD

Figure S3. Relaxed structures of (a)-(d) Kr and (e)-(h) Xe in AFM UN supercell at different sites. The teal, grey, pink, and purple balls represent uranium, nitrogen, krypton, and xenon elements, respectively.

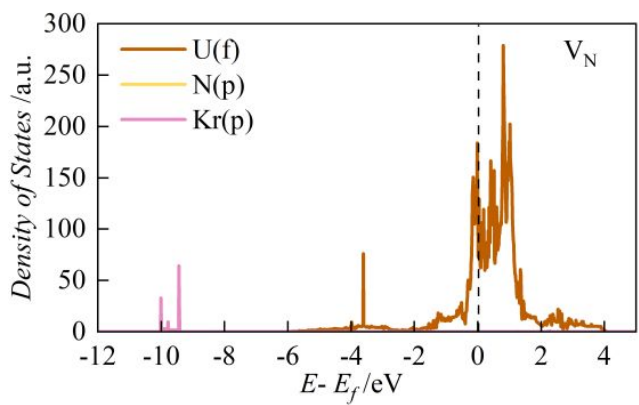

(a) $\mathrm{Kr}$ at $\mathrm{V}_{\mathrm{N}}$

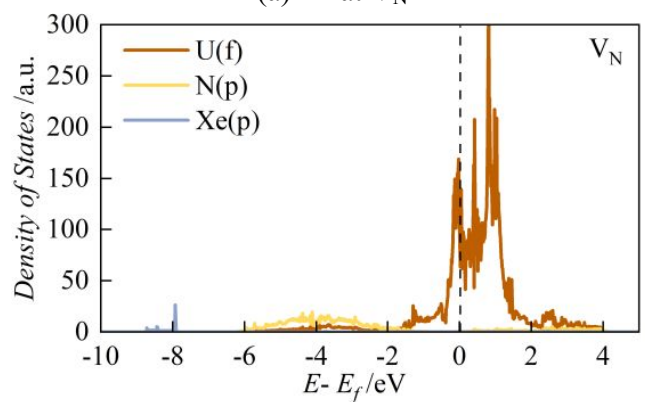

(c) $\mathrm{Xe}$ at $\mathrm{V}_{\mathrm{N}}$

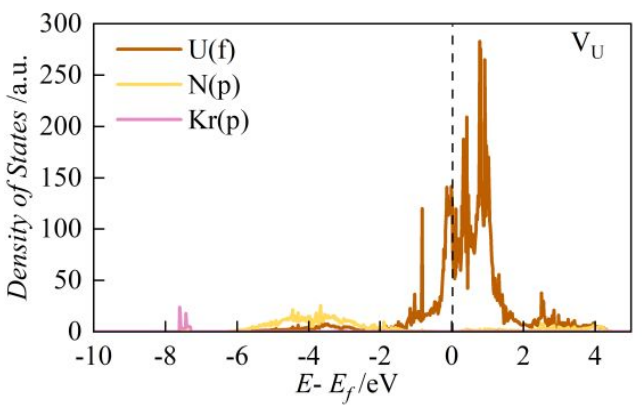

(b) $\mathrm{Kr}$ at $\mathrm{V}_{\mathrm{U}}$

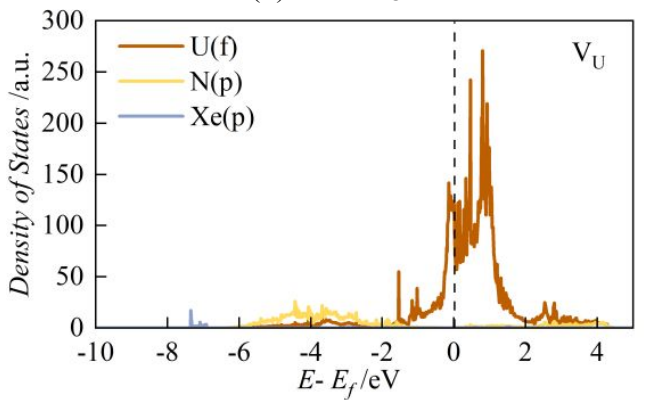

(d) $\mathrm{Xe}$ at $\mathrm{V}_{\mathrm{U}}$

Figure S4. Density of states of the $\mathrm{Kr}$ and $\mathrm{Xe}$ incorporated UN supercell. 


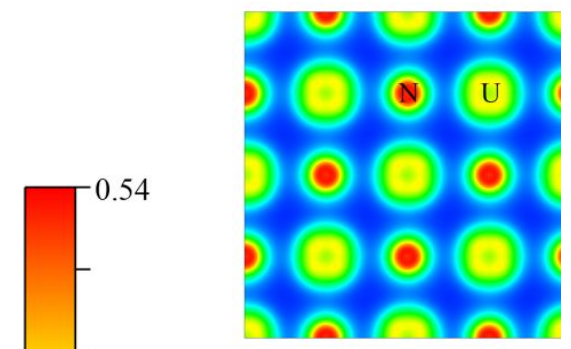

(a) UN supercell

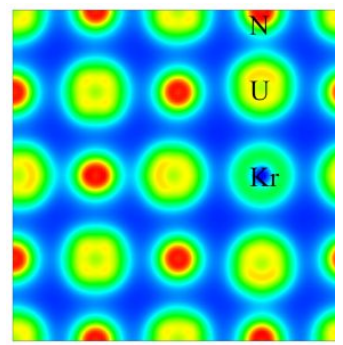

(d) $\mathrm{Kr}$ at $\mathrm{V}_{\mathrm{N}}$

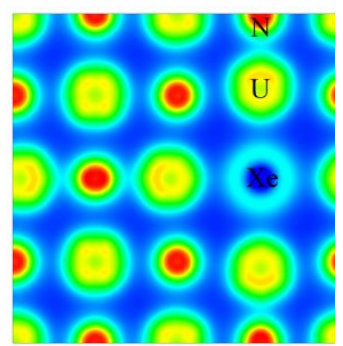

(g) Xe at $V_{N}$

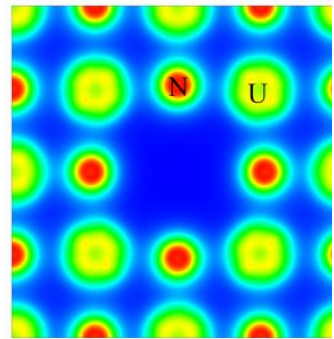

(b) $\mathrm{V}_{\mathrm{U}}$

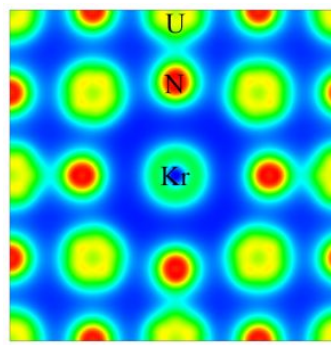

(e) $\mathrm{Kr}$ at $\mathrm{V}_{\mathrm{U}}$

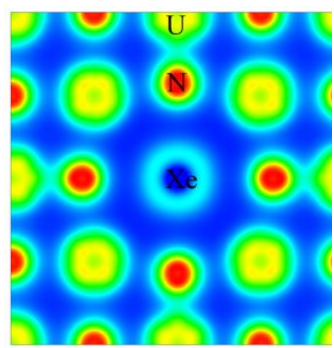

(h) $\mathrm{Xe}$ at $\mathrm{V}_{\mathrm{U}}$

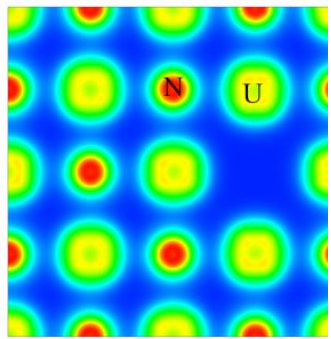

(c) $\mathrm{V}_{\mathrm{N}}$

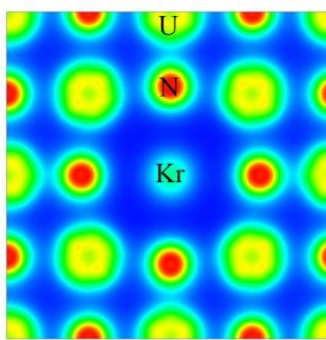

(f) $\mathrm{Kr}$ at $\mathrm{SD}$

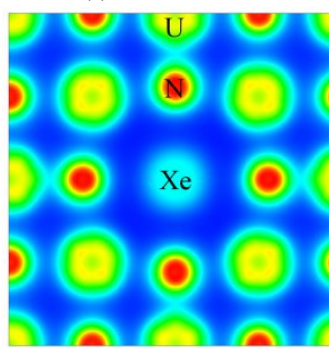

(i) $\mathrm{Xe}$ at $\mathrm{SD}$

Figure S5. Charge density distribution (a.u.) of (001) surface for (a) $U_{N}$ perfect supercell, (b) $V_{U}$ and (c) $V_{N}$ defect supercell, (d)-(f) Kr, and (g)-(i) Xe incorporated in AFM UN at different vacancy sites. 


\section{References}

(1) Klipfel, M.; Van Uffelen, P. Ab Initio Modelling of Volatile Fission Products in Uranium Mononitride. J. Nucl. Mater. 2012, 422, 137-142.

(2) Bocharov, D.; Gryaznov, D.; Zhukovskii, Y. F.; Kotomin, E. A. DFT Calculations of Point Defects on UN(001) Surface. Surf. Sci. 2011, 605, 396-400.

(3) Lan, J.-H.; Zhao, Z.-C.; Wu, Q.; Zhao, Y.-L.; Chai, Z.-F.; Shi, W.-Q. First-Principles DFT+U Modeling of Defect Behaviors in Anti-ferromagnetic Uranium Mononitride. J. Appl. Phys. 2013, 114, 223516.

(4) Kotomin, E. A.; Grimes, R. W.; Mastrikov, Y.; Ashley, N. J. Atomic Scale DFT Simulations of Point Defects in Uranium Nitride. J. Phys.: Condens. Matter 2007, 19, 106208. 